\title{
MRI and Prediction of Pathologic Complete Response in the Breast and Axilla after Neoadjuvant Chemotherapy for Breast Cancer
}

\author{
Joseph J Weber, MD1, Maxine S Jochelson, MD², Anne Eaton, MS $^{3}$, Emily C Zabor, MS ${ }^{3}$, \\ Andrea V Barrio, MD, FACS ${ }^{1}$, Mary L Gemignani, MD, FACS ${ }^{1}$, Melissa Pilewskie, MD, FACS ${ }^{1}$, \\ Kimberly J Van Zee, MD, FACS ${ }^{1}$, Monica Morrow, MD, FACS ${ }^{1}$, and Mahmoud El-Tamer, MD, \\ FACS $^{1}$ \\ ${ }^{1}$ Breast Service, Department of Surgery, Memorial Sloan Kettering Cancer Center, New York, NY \\ ${ }^{2}$ Department of Radiology, Memorial Sloan Kettering Cancer Center, New York, NY \\ ${ }^{3}$ Department of Epidemiology and Biostatistics, Memorial Sloan Kettering Cancer Center, New \\ York, NY
}

\section{Abstract}

Background-In the setting where determining extent of residual disease is key for surgical planning after neoadjuvant chemotherapy (NAC), herein we evaluate reliability of MRI in predicting pathologic complete response ( $\mathrm{pCR}$ ) of the breast primary and axillary nodes following NAC.

\begin{abstract}
Study Design-Patients who had MRI before and after NAC between 06/2014-08/2015 were identified in a prospective database following IRB approval. Post NAC-MRI of the breast and axillary nodes was correlated with residual disease on final pathology. PCR was defined as absence of invasive and in situ disease.
\end{abstract}

Results-We analyzed 129 breast cancers. Median patient age was 50.8 years (range 27.2-80.6). Tumors were HER2 amplified in 52/129 (40\%), estrogen receptor-positive/HER2-negative in $46 / 129$ (36\%) and triple negative in 31/129 (24\%), with respective pCR rates of 50\%, $11 \%$ and $29 \%$. Median tumor size pre- and post-NAC MRI were $4.1 \mathrm{~cm}$ and $1.45 \mathrm{~cm}$, respectively. MRI had a positive predictive value of $63.4 \%$ (26/41) and negative predictive value of $84.1 \%$ (74/88) for inbreast pCR.

Axillary nodes were abnormal on pre-NAC MRI in 97 cases; 65 had biopsy-confirmed metastases. The nodes normalized on post-NAC MRI in 33/65 (51\%); axillary pCR was present in 22/33

\footnotetext{
Correspondence address: Mahmoud El-Tamer, MD, Breast Service, Department of Surgery, Memorial Sloan Kettering Cancer Center 300 East $66^{\text {th }}$ Street, New York, NY 10065, eltamerm@mskcc.org.

Disclosure Information: Nothing to disclose.

Presented as a poster at the $17^{\text {th }}$ Annual Meeting of the American Society of Breast Surgeons, in Dallas, TX, April 2016.

Publisher's Disclaimer: This is a PDF file of an unedited manuscript that has been accepted for publication. As a service to our customers we are providing this early version of the manuscript. The manuscript will undergo copyediting, typesetting, and review of the resulting proof before it is published in its final citable form. Please note that during the production process errors may be discovered which could affect the content, and all legal disclaimers that apply to the journal pertain.
} 
(67\%). In 32 patients with proven nodal metastases and abnormal nodes on post-NAC MRI, 11 achieved axillary pCR. In 32 patients with normal nodes on pre- and post-NAC MRI, 6 (19\%) had metastasis on final pathology.

Conclusions-Radiologic complete response by MRI does not predict $\mathrm{pCR}$ with adequate accuracy to replace pathologic evaluation of the breast tumor and axillary nodes.

\section{INTRODUCTION}

The implementation of neoadjuvant chemotherapy (NAC) to downstage breast cancer has significantly changed surgical management of the disease regarding both the primary breast tumor and the axillary lymph nodes (ALNs). ${ }^{1,2}$ Retrospective studies have shown that a pathologic complete response (pCR) is identified in up to 33-37\% of primary tumors and $40-49 \%$ of involved ALNs depending on tumor subtype. ${ }^{3-5}$ Magnetic resonance imaging (MRI) has been demonstrated to be more accurate than physical examination, mammography, and ultrasound in assessing initial disease extent and response to NAC. ${ }^{6-8}$ Little has been published regarding the ability to detect response to NAC in the ALNs by MRI, while MRI remains the imaging modality most often used to gauge response in the breast primary tumor. ${ }^{9-14}$

Studies have shown that survival outcomes and locoregional control achieved with NAC are similar to those achieved with adjuvant chemotherapy. ${ }^{15-17}$ In practice, NAC is frequently used to convert a patient from a mastectomy to a breast-conservation candidate, to downstage axillary disease, and to provide valuable information regarding tumor response to therapy. ${ }^{18,19}$ Many studies have reported pCR can be used as a surrogate endpoint for predicting long-term clinical benefits such as disease-free survival and overall survival. ${ }^{9,20}$ There is an evolving question about the value of surgical resection of the tumor bed and ALNs in patients who have achieved a pCR. To consider omitting surgical intervention after NAC, it is paramount to accurately identify patients who achieve a pCR in the breast and the ALNs. Studies have reported on the reliability of radiologic complete response (rCR) in predicting pCR of the breast primary; however, data correlating rCR and pCR in the ALNs is limited. Here we evaluate the reliability of MRI in predicting pCR of the breast primary and ALNs following NAC.

\section{METHODS}

Following institutional review board approval, we established a HIPAA compliant, prospective database on all patients with breast cancer undergoing NAC and definitive surgical treatment at Memorial Sloan Kettering Cancer Center (MSKCC). We queried that database for patients who had a pre- and post-NAC MRI between 06/2014-08/2015. Demographic data and baseline assessments, including tumor stage and subtype, were recorded. Agreement between rCR by MRI and pCR of the breast tumor and axillary nodes on final pathology was assessed. Initial diagnosis of breast cancer was made through core biopsy. Patients received NAC as per MSKCC standard of care, including directed antihuman epidermal growth factor receptor 2 (HER2) therapy to tumors which overexpressed the HER2 oncogene. All patients underwent surgical intervention after completing chemotherapy. 
Patients underwent pre- and post-NAC MRI followed by definitive surgery. Some pretreatment MRI examinations were performed at other institutions using a variety of techniques. Those considered technically adequate were used for initial evaluation and tumor measurements. If not technically adequate, the MRI was repeated. All post-treatment MRI exams were performed at our institution with the patient prone on a 1.5T or 3.0T commercially available system (General Electric Medical Systems, Milwaukee, WI). Both breasts were imaged simultaneously using VIBRANT. The standard exam included a localizing sequence followed by axial fat-suppressed T2-weighted and T1-weighted sequences. Diffusion-weighted imaging was also performed. A T1-weighted threedimensional, fat-suppressed, gradient-echo sequence was performed before, and three times after, an intravenous rapid bolus injection of $0.1 \mathrm{mmol} / \mathrm{L}$ of gadopentetate dimeglumine (Magnevist, Berlex, and Wayne, NJ) per kilogram of body weight followed by a saline bolus. Images were acquired in an axial projection with sagittal reformation. Section thickness was $0.1 \mathrm{~cm}$ with no gap and a minimum matrix of 256x256. Unenhanced images were subtracted from the contrast-enhanced images on a pixel-by-pixel basis producing one subtracted postcontrast subtraction sequence. Maximum intensity projection images were created using the first postcontrast sequence and the first postcontrast subtracted data.

All pre- and post-NAC breast MRIs were secondarily reviewed by an experienced breast radiologist (MJ) blinded to the final pathology. Changes in the enhancing tumor size and nodal response from pre- to post-NAC MRI examinations were documented. The classification of rCR in the breast was applied when all available MRI images revealed no enhancement in the tumor bed. The axillary nodes were reported as abnormal on MRI both before and after chemotherapy when they had a thickened or irregular cortex $(>4 \mathrm{~mm})$, round shape, and/or loss of the fatty hilum. Enhancement of the axillary nodes on MRI was not considered as a criterion of malignancy, as benign nodes normally enhance.

The expression status of the estrogen receptor (ER), progesterone receptor (PR), and HER2 was determined from histopathologic reports of core biopsies performed before chemotherapy. Hormone receptor positivity was defined as $>1 \%$ of cells staining for ER or PR. Tumors with HER2 scores of 3+ (strong homogeneous staining) were considered positive. In tumors with $2+$ scores (moderate complete membrane staining in $\geq 10 \%$ of tumor cells), in-situ hybridization was used to determine HER2 amplification.

PCR in the breast was defined as the absence of both invasive and in situ disease. All lymph nodes (LNs) were evaluated for evidence of metastatic malignant cells by using hematoxylin and eosin stains. Immunohistochemical stains were limited to cases in which axillary nodal metastasis was questionable. The absence of tumor cells on final pathology in patients with proven nodal metastasis prior to NAC was considered a pCR in the LNs. Positive predictive value (PPV) was defined as the number of patients with both an $\mathrm{rCR}$ and a $\mathrm{pCR}$, divided by the total number of patients with an rCR. Negative predictive value (NPV) was defined as the number of patients without an rCR who also did not have a pCR, divided by the total number of patients without an $\mathrm{rCR}$ 


\section{RESULTS}

From 06/2014-08/2015 we identified 128 patients with 129 breast cancers who received NAC followed by definitive breast surgery at MSKCC, and had a pre- and post-NAC breast MRI.

Table 1 describes patient demographics. Patient population median age was 50.8 years (range 27.2-80.6). The most frequent tumor subtype had overexpression of HER2 (52/129; $40.3 \%)$, the majority of which were ER positive (41/52). Approximately one-quarter of the study population had triple-negative tumors $(31 / 129 ; 24.0 \%)$; the remainder had ERpositive/HER2-negative tumors $(46 / 129 ; 35.7 \%)$. The majority of women presented with T2 tumors (60.5\%). At initial presentation, $123(95.4 \%)$ breast primaries were palpable. Following NAC, 83/123 tumors were no longer palpable for an in-breast complete clinical response rate by physical examination of $67 \%$.

The median size of the primary tumor on pre-NAC MRI was $4.1 \mathrm{~cm}$ (range 0.7-15.0). Following NAC, median size of the primary tumor on MRI was $1.5 \mathrm{~cm}$ (range 0.0-9.0). Forty-five percent of patients underwent mastectomy; 55\% underwent breast-conserving surgery.

Table 2 lists $\mathrm{rCR}$ and $\mathrm{pCR}$ rates in the breast. The overall primary tumor $\mathrm{pCR}$ rate following NAC was $31 \%$. The highest rCR and pCR rates were seen in the HER2-amplified tumors: $51.9 \%$ and $50 \%$, respectively.

Table 3 describes the correlation of rCR by MRI and pCR in the breast. The rCR by MRI correctly predicted pCR in $26 / 41$ cases for a PPV of $63.4 \%$. Residual MRI abnormality correlated with residual disease on histology in $74 / 88$ cases, for an NPV of $84.1 \%$.

Axillary surgery included sentinel lymph node biopsy (SLNB) only in 74 cases, SLNB was followed by completion ALN dissection (ALND) in 25, and initial ALND was performed in 30. Thirty-two had normal axillary nodes on pre- and post-NAC MRI; of those, 26 (81.2\%) had negative nodes and $6(18.8 \%)$ had nodal metastasis on final pathology. In 50 that had abnormal nodes which normalized on MRI post-NAC, final pathology showed no metastasis in $34(68 \%)$ (Table 4).

Sixty-five patients with abnormal nodes seen on pretreatment MRI had biopsy-proven metastases (Table 5); post-NAC MRI exhibited normal nodes in approximately half, 33/65 (50.8\%). In cases where axillary rCR was achieved, axillary pCR was present in 22/33 (PPV $66.7 \%$ ). In 32 cases, the axillary nodes remained abnormal on post-NAC MRI, 21 of whom had residual disease on final pathology (NPV 65.6\%).

\section{DISCUSSION}

Surgical management of breast cancer has been significantly impacted by the increasing use of NAC. Breast MRI is widely used to evaluate extent of disease before treatment and assess response to NAC, and has been reported to more reliably predict response of the primary tumor when compared to clinical exam and other imaging modalities, such as 
mammography or ultrasound. ${ }^{21}$ With modern chemotherapy and the addition of targeted anti-HER 2 treatment, the in-breast and axillary pCR rates have significantly increased, reaching up to $50 \% .^{3-5}$ The role of surgical resection in patients who have achieved an $\mathrm{rCR}$ is for confirmation of the completeness of response, and, accordingly, therapeutic value of surgery has been questioned. However, to consider omitting surgical intervention following NAC, patients who not only have achieved a pCR in the breast but also in the axillary nodes must be accurately identified. Here we explored the ability of MRI to predict pCR in both the primary tumor and ALNs following NAC.

The overall breast pCR rate in our study was $31 \%$ : in keeping with rates reported in other modern studies; in-breast pCR rates were $34 \%$ in ACOSOG Z107122 and 26\% in NSABP B-27. ${ }^{16}$ The nodal $\mathrm{pCR}$ rate among all 65 biopsy-proven node-positive patients with abnormal nodal imaging by MRI was 50.8\%, slightly higher than the $41.1 \%$ ACOSOG Z1071 overall axillary pCR rate. ${ }^{22}$

Our primary focus was to determine the diagnostic accuracy of radiologic response with pathologic assessment as the gold standard. In our data analysis, PPV was defined as the number of patients with both an rCR and a pCR, divided by the total number of patients with an rCR. NPV was defined as the number of patients without an rCR who also did not have a $\mathrm{pCR}$, divided by the total number of patients without an $\mathrm{rCR}$. With respect to the primary breast tumor, we found that the PPV and NPV of MRI to predict pCR were $63 \%$ and $84 \%$, respectively. A systematic review to assess the role of MRI in predicting pCR after NAC was performed by Lobbes. By our definition of PPV and NPV, they found a range of PPV from 71-100\% and NPV from $47-73 \% .^{21}$ Their analysis found that both overestimation and underestimation of residual disease extent can be observed, and cancer subtype and treatment regimen may influence MRI accuracy. Additionally, there is variability among institutions with MRI image acquisition protocols, and differences may be found due to positioning of the patient, body habitus, as well as MRI hardware and software. Our study has shown that post-NAC-MRI was accurate in predicting pCR in $63.4 \%$ of the cases (PPV). Hence, $37 \%$ of patients who had achieved a complete response by MRI after NAC had residual disease. In order to consider foregoing surgical resection of the tumor bed, we have to rely on a test that allows us to positively predict pCR in more than $95 \%$ of the cases.

Gonzalez-Cortijo showed a $100 \%$ NPV of MRI for predicting primary breast pCR following NAC among all tumor subtypes. ${ }^{23}$ In their 75-patient series, they used an 8-minute delay following contrast injection to classify MRI enhancement. Per our institutional protocol, once contrast injection is complete, the patient is moved back immediately into the scanner to resume imaging. It is unclear whether waiting additional time following contrast injection would impact MRI NPV. Importantly, Gonzalez-Cortijo classified a pCR as no invasive tumor only, even if residual DCIS was found on final surgical pathology, which likely impacts the accuracy of MRI in assessing pCR. In our series, we defined a pCR when there was no invasive disease or DCIS in the final surgical specimen.

Although MRI is commonly used to identify residual disease with respect to the primary breast tumor, there are limited data in predicting pCR within the ALNs. As opposed to the objective definition of rCR in the breast, which is the absence of any enhancement, 
definition of rCR in the LNs is difficult to measure due to normal variations in nodal size and architecture among patients, as well as the inability to use enhancement as a criteria for malignancy in the nodes. Exact cortical measurement on MRI may change depending on positioning, and morphologic nodal change from round to oval may also be affected by patient position. ${ }^{9}$ Additionally, there are other technical aspects which may affect MRI analysis of LN response, including respiratory motion artifact from the chest wall and potential false identification of LNs from adjacent vascular structures. ${ }^{9,24}$ Hieken used perinodal enhancement to gauge suspicious adenopathy. ${ }^{9}$ They demonstrated that ultrasound was the most sensitive method of evaluating the post-NAC axilla, with respective sensitivities of $70 \%$ by ultrasound, $63 \%$ by position emission tomography (PET), and $61 \%$ by MRI. It was also reported that PET was the most accurate at $72 \%$, compared to accuracy of ultrasound at $65 \%$ and MRI at $60 \%$. From the revised RECIST guidelines, nodes that shrink to $<10 \mathrm{~mm}$ short axis were considered normal on post-NAC imaging. ${ }^{11}$ Morphology of the LNs has also been used to predict malignancy, such as a round shape to the node, loss of the fatty hilum, and thickened cortices. ${ }^{25}$

Javid found that in patients who were node positive prior to NAC, MRI could predict with moderate sensitivity $(85.7 \%)$ and specificity (89\%) whether residual nodal disease was present. Based on our definition of predictive values, they found MRI could predict pCR in the axilla with a PPV and NPV of $80.9 \%$ and $92 \%$, respectively. ${ }^{26}$ However, a recent prospective analysis by Mattingly showed a PPV of 50\% and NPV of $66.7 \%$ for pCR in the ALNs when evaluated by post-NAC MRI by our definition of predictive values. ${ }^{27}$ In our dataset, cases with biopsy-proven nodal metastasis, an rCR on post-NAC MRI had a PPV of $66.7 \%$ and an NPV of $65.6 \%$ in predicting a pCR on final excision. Based on these findings, MRI alone does not obviate the need for surgical nodal staging following NAC.

The accuracy of MRI for assessing response in the breast has been shown to vary based on ER, PR, and HER2 status. ${ }^{6,28}$ It is well established that pCR rates differ by tumor subtype, with higher pCR rates in HER2-overexpressing and triple-negative breast cancers, and the lowest rates seen in hormone receptor-positive cancers. ${ }^{5,29-31}$ De Los Santos demonstrated an overall accuracy of $74 \%$ with respect to the ability of MRI to predict pCR, but noted the greatest NPVs in patients with triple-negative (60\%) and HER2-positive disease (62\%), likely due to an excellent response in those patients. ${ }^{7}$ From our data, the greatest pCR in the breast primary was seen in HER2-amplified tumors (50\%) and in triple-negative tumors (29\%). The ER-positive, HER2-negative patients had a pCR of $10.9 \%$, the lowest rate in our population, similar to other published studies.

Our report has certain limitations. This is a single-institution retrospective study with a small number of patients. All MRI imaging was performed based on our institutional protocol, hence the results may not be generalizable. Additionally, this was a relatively small sample size; following subgroup analysis, potentially, there are underrepresented populations which may limit generalizability of the results. 


\section{CONCLUSIONS}

Here we demonstrate resolution of disease on MRI is predictive of pathologic resolution of the breast primary tumor and in biopsy-proven ALNs in only $63 \%$ and $68 \%$ of cases, respectively. These data confirm that despite rCR by MRI, surgical resection with pathologic evaluation of the breast tissue and ALNs remains necessary to reliably confirm pCR and ensure resection of microscopic residual disease.

\section{Acknowledgments}

Support: The preparation of this manuscript was funded in part by NIH/NCI Cancer Center Support Grant No. P30 CA008748 to Memorial Sloan Kettering Cancer Center.

The authors thank Michelle Stempel, Imelda Burgan, Deborah Capko, MD, Hiram S Cody, MD, Alexandra S Heerdt, MD, George Plitas, MD, Virgilio Sacchini, MD, and Lisa M Sclafani, MD for their clinical volume, database assistance, and critical review contributions to this article.

\section{References}

1. Bear HD, Anderson S, Brown A, et al. The effect on tumor response of adding sequential preoperative docetaxel to preoperative doxorubicin and cyclophosphamide: preliminary results from National Surgical Adjuvant Breast and Bowel Project Protocol B-27. J Clin Oncol. 2003; 21:416574. [PubMed: 14559892]

2. Fisher B, Bryant J, Wolmark N, et al. Effect of preoperative chemotherapy on the outcome of women with operable breast cancer. J Clin Oncol. 1998; 16:2672-85. [PubMed: 9704717]

3. Hennessy BT, Hortobagyi GN, Rouzier R, et al. Outcome after pathologic complete eradication of cytologically proven breast cancer axillary node metastases following primary chemotherapy. J Clin Oncol. 2005; 23(36):9304-93. [PubMed: 16361629]

4. von Minckwitz G, Untch M, Blohmer JU, et al. Definition and impact of pathologic complete response on prognosis after neoadjuvant chemotherapy in various intrinsic breast cancer subtypes. J Clin Oncol. 2012; 30:1796-1804. [PubMed: 22508812]

5. Dominici LS, Negron Gonzalez VM, Buzdar AU, et al. Cytologically proven axillary lymph node metastases are eradicated in patients receiving preoperative chemotherapy with concurrent trastuzumab for HER2-positive breast cancer. Cancer. 2010; 116:2884-89. [PubMed: 20564395]

6. Loo CE, Straver ME, Rodenhuis S, et al. Magnetic resonance imaging response monitoring of breast cancer during neoadjuvant chemotherapy: relevance of breast cancer subtype. J Clin Oncol. 2011; 29:660-6. [PubMed: 21220595]

7. De Los Santos JF, Cantor A, Amos KD, et al. Magnetic resonance imaging as a predictor of pathologic response in patients treated with neoadjuvant systemic therapy for operative breast cancer. Translation Breast Cancer Research Consortium trial 017. Cancer. 2013; 119(10):1776-83. [PubMed: 23436342]

8. Chen JH, Bahri S, Mehta RS, et al. Breast cancer: evaluation of response to neoadjuvant chemotherapy with 3. 0-T. MR imaging Radiology. 2011; 261(3):735-43. [PubMed: 21878615]

9. Hieken TJ, Boughey JC, Jones KN, et al. Imaging response and residual metastatic axillary lymph node disease after neoadjuvant chemotherapy for primary breast cancer. Ann Surg Oncol. 2013; 20:3199-3204. [PubMed: 23846781]

10. Cameron DA, Anderson ED, Levack P, et al. Primary systemic therapy for operable breast cancer--10-year survival data after chemotherapy and hormone therapy. Br J Cancer. 1997; 76:1099-1105. [PubMed: 9376273]

11. Eisenhauer EA, Therasses P, Bogaerts J, et al. New response evaluation in solid tumours: Revised RECIST guideline (version 1. 1). Eur J Cancer. 2009; 45:228-47. [PubMed: 19097774]

12. Abraham DC, Jones RC, Jones SE, et al. Evaluation of neoadjuvant chemotherapeutic response of locally advanced breast cancer by magnetic resonance imaging. Cancer. 1996; 78:91-100. [PubMed: 8646731] 
13. Balu-Maestro C, Chapellier C, Bleuse A, et al. Imaging in evaluation of response to neoadjuvant breast cancer treatment benefits of MRI. Breast Cancer Res Treat. 2002; 72:145-52. [PubMed: 12038705]

14. Weatherall PT, Evans GF, Metzger GJ, et al. MRI vs. histologic measurement of breast cancer following chemotherapy: comparison with x-ray mammography and palpation. J Magn Reson Imaging. 2001; 13:868-75. [PubMed: 11382946]

15. Gralow JR, Burstein HJ, Wood W, et al. Preoperative therapy in invasive breast cancer: pathologic assessment and systemic therapy issues in operable disease. J Clin Oncol. 2008; 26:814-19. [PubMed: 18258991]

16. Rastogi P, Anderson SJ, Bear HD, et al. Preoperative chemotherapy: updates of National Surgical Adjuvant Breast and Bowel Project Protocols B-18 and B-27. J Clin Oncol. 2008; 26:778-85. [PubMed: 18258986]

17. van der Hage JA, van de Velde CJ, Julien JP, et al. Preoperative chemotherapy in primary operable breast cancer: results from the European Organization for Research and Treatment of Cancer trial 10902. J Clin Oncol. 2001; 19:4224-37. [PubMed: 11709566]

18. Buchholz TA, Lehman CD, Harris JR, et al. Statement of the science concerning locoregional treatments after preoperative chemotherapy for breast cancer: a National Cancer Institute conference. J Clin Oncol. 2008; 26:791-7. [PubMed: 18258988]

19. von Minckwitz G, Blohmer JU, Costa SD, et al. Response-guided neoadjuvant chemotherapy for breast cancer. J Clin Oncol. 2013; 31:3623-30. [PubMed: 24002511]

20. Liedtke C, Mazouni C, Hess KR, et al. Response to neoadjuvant therapy and long-term survival in patients with triple-negative breast cancer. J Clin Oncol. 2008; 26:1275-81. [PubMed: 18250347]

21. Lobbes M, Prevos R, Smidt M, et al. The role of magnetic resonance imaging in assessing residual disease and pathologic complete response in breast cancer patients receiving neoadjuvant chemotherapy: a systematic review. Insights Imaging. 2013; 2:163-75.

22. Boughey JC, Suman VJ, Mittendorf EA, et al. Sentinel lymph node surgery after neoadjuvant chemotherapy in patients with node-positive breast cancer: The ACOSOG Z1071 (Alliance) Clinical Trial. JAMA. 2013; 310:1455-61. [PubMed: 24101169]

23. Gonzalez-Cortijo L, Hornedo J, Sainz de la Cuesta R, et al. Magnetic resonance imaging evaluation of pathologic complete response in different breast cancer subtyes after neoadjuvant chemotherapy. J Clin Oncol. 2012; 30(suppl) abstr 1111.

24. Schipper RJ, Moossdorff M, Beets-Tan RGH, et al. Noninvasive nodal restaging in clinically node positive breast cancer patients after neoadjuvant systemic therapy: A systematic review. Euro $\mathrm{J}$ Radiol. 2015; 84(1):41-7.

25. Rahbar H, Conlin JL, Parsian S, et al. Suspicious axillary lymph nodes identified on clinical breast MRI in patients newly diagnosed with breast cancer: can quantitative features improve discrimination of malignant from benign? Acad Radiol. 2015; 22:430-8. [PubMed: 25491740]

26. Javid S, Segara D, Lotfi P, et al. Can breast MRI predict axillary lymph node metastasis in women undergoing neoadjuvant chemotherapy. Ann Surg Oncol. 2010; 17:1841-6. [PubMed: 20143266]

27. Mattingly AE, Mooney B, Hui-Yi L, et al. Magnetic resonance imaging for axillary breast cancer metastasis in the neoadjuvant setting: a prospective study. Clin Breast Cancer. 2016 (In press).

28. Chen JH, Feig B, Agrawal G, et al. MRI evaluation of pathologically complete response and residual tumors in breast cancer after neoadjuvant chemotherapy. Cancer. 2008; 112:17-26. [PubMed: 18000804]

29. Connor CA, Kimler BF, Mammen JMV, et al. Impact of neoadjuvant chemotherapy on axillary nodal involvement in patients with clinically node negative triple negative breast cancer. J Surg Oncol. 2015; 111(2):198-202. [PubMed: 25266871]

30. McVeigh TP, Al-Azawi D, Kearney DE, et al. Assessing the impact of neoadjuvant chemotherapy on the management of the breast and axilla in breast cancer. Clin Breast Cancer. 2014; 14(1):20-5. [PubMed: 24157259]

31. Park S, Park JM, Cho JH, et al. Sentinel lymph node biopsy after neoadjuvant chemotherapy in patients with cytologically proven node-positive breast cancer at diagnosis. Ann Surg Oncol. 2013; 20(9):2858-65. [PubMed: 23645483] 


\section{Table 1}

Patient Demographics of the Entire Population

\begin{tabular}{|c|c|}
\hline Variable & Data $(n=129)$ \\
\hline Age at diagnosis, y, median (range) & $50.8(27.2-80.6)$ \\
\hline \multicolumn{2}{|l|}{ Clinical T stage, $\mathrm{n}(\%)$} \\
\hline 1 & $12(9.3)$ \\
\hline 2 & $78(60.5)$ \\
\hline 3 & $31(24.0)$ \\
\hline 4 & $8(6.2)$ \\
\hline \multicolumn{2}{|l|}{ Clinical N stage, $\mathrm{n}(\%)$} \\
\hline 0 & $46(35.7)$ \\
\hline 1 & $68(52.7)$ \\
\hline 2 & $10(7.8)$ \\
\hline 3 & $5(3.9)$ \\
\hline \multicolumn{2}{|l|}{ Clinical stage, n (\%) } \\
\hline 1a & $3(2.3)$ \\
\hline $2 \mathrm{a}$ & $38(29.5)$ \\
\hline $2 b$ & $46(35.7)$ \\
\hline $3 \mathrm{a}$ & $30(23.3)$ \\
\hline $3 b$ & $7(5.4)$ \\
\hline $3 c$ & $5(3.9)$ \\
\hline \multicolumn{2}{|l|}{ Receptor status, n (\%) } \\
\hline ER+ HER2- & $46(35.7)$ \\
\hline ER+ HER2+ & $41(31.8)$ \\
\hline ER-HER2+ & $11(8.5)$ \\
\hline ER- HER2- & $31(24.0)$ \\
\hline
\end{tabular}

T, tumor; N, nodal; ER, estrogen receptor; HER2, human epidermal growth factor 2 


\section{Table 2}

Breast Radiologic Complete Response and Pathologic Complete Response Rates for the Total Study Population and Stratified by Receptor Status

\begin{tabular}{|l|c|c|}
\hline Variable & Primary breast $\mathbf{r C R}^{*}, \mathbf{n}(\boldsymbol{\%})$ & Primary breast $\mathbf{p C R} \mathbf{C R}^{\dagger}, \mathbf{n}(\boldsymbol{\%})$ \\
\hline Total study population $(\mathrm{n}=129)$ & $41(31.8)$ & $40(31.0)$ \\
\hline Receptor status & & \\
\hline ER+ HER2- $(\mathrm{n}=45)$ & $4(8.9)$ & $4(8.9)$ \\
\hline ER+ HER2+ $(\mathrm{n}=40)$ & $22(55.0)$ & $19(47.5)$ \\
\hline ER- HER2+ $(\mathrm{n}=12)$ & $5(41.7)$ & $7(58.3)$ \\
\hline ER- HER2- $(\mathrm{n}=32)$ & $10(31.3)$ & $10(31.3)$ \\
\hline
\end{tabular}

*CR defined as no residual enhancement on post-neoadjuvant chemotherapy MRI at site of biopsy proven breast cancer compared to normal background tissue

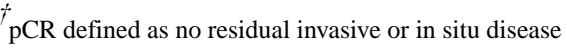

$\mathrm{rCR}$, radiologic complete response; pCR, pathologic complete response; ER, estrogen receptor; HER2, human epidermal growth factor 2 
Table 3

Correlation of Radiologic Complete Response by Post- Neoadjuvant Chemotherapy MRI with Pathologic Complete Response in the Breast

\begin{tabular}{|c|c|c|c|}
\hline Variable & pCR in breast & No pCR in breast & Total \\
\hline rCR in breast, $\mathrm{n}(\%)$ & $26(63.4)^{*}$ & 15 (36.6) & 41 \\
\hline No rCR in breast, $\mathrm{n}(\%)$ & $14(15.9)$ & $74(84.1)^{\dagger}$ & 88 \\
\hline Total, $\mathrm{n}$ & 40 & 89 & 129 \\
\hline
\end{tabular}

rCR, radiologic complete response; pCR, pathologic complete response 


\section{Table 4}

Correlation of Pre- and Post- Neoadjuvant Chemotherapy Axillary Nodal Status on MRI with Final Pathology Finding for Entire Population ( $\mathrm{N}=129)$

\begin{tabular}{|l|c|c|}
\hline \multirow{2}{*}{ Axillary nodal status on MRI } & \multicolumn{2}{|c|}{ Final pathology of axillary nodes, $\mathbf{n}(\%)$} \\
\cline { 2 - 3 } & No metastasis $(\mathbf{n}=\mathbf{8 0})$ & Metastasis $(\mathbf{n}=\mathbf{4 9})$ \\
\hline Normal pre- and post-NAC $(\mathrm{n}=32)$ & $26(81.2)$ & $6(18.8)$ \\
\hline Abnormal pre-NAC, Normal post-NAC $(\mathrm{n}=50)$ & $34(68)$ & $16(32)$ \\
\hline Abnormal pre- and post-NAC $(\mathrm{n}=47)$ & $20(42.6)$ & $27(57.4)$ \\
\hline
\end{tabular}

pCR, pathologic complete response; NAC, neoadjuvant chemotherapy; MRI, magnetic resonance imaging 
Table 5

Correlation of Pre- and Post- Neoadjuvant Chemotherapy Axillary Nodal Status on MRI with Pathologic Complete Response in Patients with Biopsy-Proven Node-Positive Disease $(\mathrm{N}=65)$

\begin{tabular}{|l|c|c|}
\hline \multirow{2}{*}{ Axillary nodal status on MRI* } & \multicolumn{2}{|c|}{ Final pathology of axillary nodes, $\mathbf{n}(\%)$} \\
\cline { 2 - 3 } & Nodal $\mathbf{p C R}^{\dagger}(\mathbf{n}=\mathbf{3 3})$ & No nodal $\mathbf{~} \mathbf{C R}^{\dagger}(\mathbf{n}=\mathbf{3 2})$ \\
\hline Abnormal pre-NAC, normal post-NAC $(\mathrm{n}=33)$ & $22(66.7){ }^{\dagger}$ & $11(33.3)$ \\
\hline Abnormal pre- and post-NAC $(\mathrm{n}=32)$ & $11(34.4)$ & $21(65.6)^{\xi}$ \\
\hline
\end{tabular}

* Cases with pre-NAC biopsy proven malignancy but had normal nodes on MRI pre- and post-NAC were not included in the tables ${ }^{\dagger} \mathrm{pCR}$ defined as no residual disease in the axillary nodes

tPost- neoadjuvant chemotherapy MRI negative predictive value

$\mathcal{S}_{\text {Post- neoadjuvant chemotherapy MRI positive predictive value }}$

pCR, pathologic complete response; NAC, neoadjuvant chemotherapy; MRI, magnetic resonance imaging 